\title{
Differential Forms \\ (Open Mathematics Knowledge Base)
}

\author{
Open Mathematics Collaboration*†
}

July 21, 2020

\begin{abstract}
We present a list of mathematical results about differential forms for future implementation in a digital Open Mathematics Knowledge Base.
\end{abstract}

keywords: differential geometry, pure mathematics, knowledge base

The most updated version of this paper is available at https://osf.io/g7uqb/download

\section{Introduction}
A. $11-3$
B. OMKB = Open Mathematics Knowledge Base (see 44)
C. This article is constantly being updated.
D. Differential Forms $(\mathrm{OMKB})=56$ mathematical entries (30 pages)
E. 1 entry = notation or definition or proposition or theorem

*All authors with their affiliations appear at the end of this paper.

†Corresponding author: alvaroyucra@uft.edu.br | Join the Open Mathematics Collaboration 


\section{Overview}

F. 5

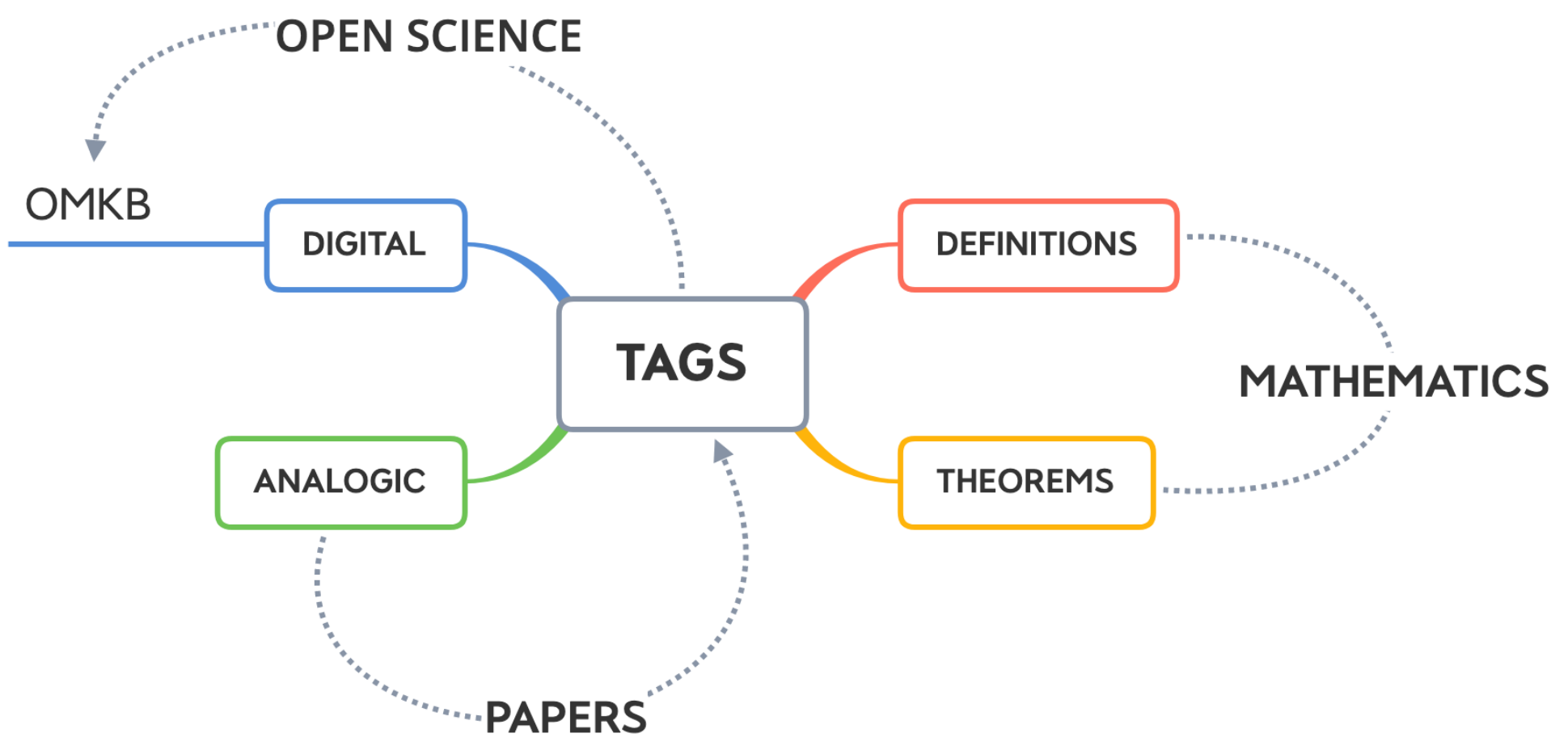




\section{Important: guidelines for advanced search}

G. In order to maximize the efficiency while using this document, note the following guidelines for advanced search in Acrobat Reader.

H. Acrobat Reader $\rightarrow$ Preferences $\rightarrow$ Search $\rightarrow$ Range of words for proximity searches $\approx 10$

I. Acrobat Reader $\rightarrow$ open the search window $\rightarrow$ advanced settings $\rightarrow$ show more options $\rightarrow$ select a folder where the PDF is located $\rightarrow$ check the proximity box $\rightarrow$ choose match all of the words 


\section{\#alternating projection \#definition \#notation}

1. The alternating projection is a linear map Alt $: L_{r}(V) \rightarrow A_{r}(V)$

2. $\operatorname{Alt}(\psi)=$ alternating projection of $\psi \in L_{r}(V)$

3. $\operatorname{Alt}(\psi)\left(u_{1}, \ldots, u_{r}\right)=\frac{1}{r !} \sum_{\sigma \in P_{r}} \operatorname{sgn} \sigma \cdot\left(u_{\sigma(1)}, \ldots, u_{\sigma(r)}\right)$ for any vectors $u_{1}, \ldots, u_{r} \in V$ 


\section{\#alternating projection \#properties}

4. $\operatorname{Alt}(\psi) \in A_{r}(V)$, for all $\psi \in L_{r}(V)$

5. If $\psi \in A_{r}(V) \Rightarrow \operatorname{Alt}(\psi)=\psi$

6. $\operatorname{Alt}(\operatorname{Alt}(\psi))=\operatorname{Alt}(\psi)$, for all $\psi \in L_{r}(V)$ 
\# anticommutativity \# exterior product \#linear forms

7. $\psi \wedge \xi=(-1)^{r s} \xi \wedge \psi$, where $\psi \in A_{r}(V)$ and $\xi \in A_{s}(V)$ (anticommutativity)

8. $\operatorname{dim} A_{n}(V)=1$

9. If $r>n \Rightarrow A_{r}(V)=\{0\}$ 


\section{\# antisymmetric \#definition \# notation \#r-linear form}

10. A map $\psi \in L_{r}(V)$ is antisymmetric if

$$
\psi\left(u_{1}, \ldots, u_{i}, \ldots, u_{j}, \ldots, u_{r}\right)=-\psi\left(u_{1}, \ldots, u_{j}, \ldots, u_{i}, \ldots, u_{r}\right)
$$

11. $\psi \in L_{r}(V)$ is a $r$-linear form $\Leftrightarrow \psi$ é antisymmetric

12. $A_{r}(V)=$ set of all $r$-linear forms

13. $A_{r}(V) \subset L_{r}(V)$ is a vector subspace

14. $A_{0}(V)=\mathbb{R}$

15. $A_{1}(V)=V^{*}$ 


\section{\#basis \#coordinate functions \#definition}

\section{\#differential forms \#r-linear form}

16. (a) $I=\left\{i_{1}<\ldots<i_{r}\right\}=r$-list

(b) $\left\{d w_{I}(p)=d w_{i_{1}}(p) \wedge \ldots \wedge d w_{i_{r}}(p): I\right.$ is a $r$-list $\}$ forms a basis for $A_{r}\left(T_{p} M\right)$

17. (a) If $\psi$ is a differential form of degree $r \Rightarrow \psi=\sum_{I} a_{I}(p) d w_{I}(p)$ for all $p \in X(W)$

(b) $a_{I}: X(W) \rightarrow \mathbb{R}=$ coordinate functions of $\psi$ in relation to $X$ 
\#basis \#definition \#tensor product

18. If $\left\{g_{1}, \ldots, g_{n}\right\}$ is a basis for $V^{*} \Rightarrow\left\{g_{i_{1}} \otimes g_{i_{2}} \otimes \ldots \otimes g_{i_{r}}: 1 \leq i_{1} \leq \ldots i_{r} \leq\right.$ $n\}$ is a basis for $L_{r}(V)$ 
\#basis \# exterior product \#linear forms \#sequence of indices

19. If $\left\{f_{1}, \ldots, f_{n}\right\}$ is any basis for $V^{*}$ and $I=\left(i_{1}, \ldots, i_{r}\right)$ a sequence of indices of the set $\{1,2, \ldots, n\} \Rightarrow f_{I}=f_{i_{1}} \wedge \ldots \wedge f_{i_{r}} \in A_{r}(V)$

20. $I=\left\{i_{1}<\ldots<i_{r}\right\}$ is called a $r$-list

21. If $\left\{f_{1}, \ldots, f_{n}\right\}$ is any basis for $V^{*} \Rightarrow\left\{f_{I}: I\right.$ is a $r$-list $\}$ forms a basis for $A_{r}(V)$

22. $\operatorname{dim} A_{r}(V)=\frac{n !}{r !(n-r) !}$ 
\# coordinate functions \#definition \#differentiable \#differential forms \#notation

23. If $a_{I}$ are differentiable in each coordinate neighborhood of $M$ for all $r$-list $I \Rightarrow$ the differential form of degree $r, \psi$, is differentiable

24. $\Omega^{r}(M)=$ set of differential forms of degree $r$ that are differentiable in $M$ 


\section{\#definition \#differentiable \#differential forms} \# exterior product \#wedge product

25. (a) Let $\beta \in \Omega^{r}(M)$ and let $\psi \in \Omega^{s}(M)$

(b) $\beta \wedge \psi=$ wedge product of $\beta$ and $\psi$. This is a differential form of degree $r+s$, defined by

$$
\beta \wedge \psi(p)=\beta(p) \wedge \psi(p)
$$

for all $p \in M$

(c) $\beta(p) \wedge \psi(p)=$ exterior product of the linear forms $\beta(p)$ and $\psi(p)$ 


\section{\#definition \#differentiable \#differential forms}

\section{\#pullback \#smooth map}

26. (a) Let $f: M \rightarrow N$ be a smooth map between two surfaces, and $\psi \in \Omega^{r}(N)$

(b) $f^{*} \psi \subset \Omega^{r}(M)=$ pullback of $\psi$ through $f$, defined by

$$
f^{*} \psi(p)\left(u_{1}, \ldots, u_{r}\right)=\psi(f(p))\left(d f(p) \cdot u_{1}, \ldots, d f(p) \cdot u_{r}\right)
$$

for any $p \in M$ and $u_{1}, \ldots, u_{r} \in T_{p} M$

27. If $\left.g \in \Omega^{0}(N)\right) \Rightarrow g \in C^{\infty}(N)$ and $f^{*} g=g \circ f: M \rightarrow \mathbb{R}$ 


\section{\#definition \#differential forms \#dual basis}

\section{\#parametrization \#tangent space}

28. (a) $X: W \rightarrow X(W)=$ parametrization of $M, W \subset \mathbb{R}^{n}$ is an open set

(b) For each $p=X(x) \in X(W) \subset M,\left\{d X(x) \cdot e_{1}, \ldots, d X(x) \cdot e_{n}\right\}$ is a basis for $T_{p} M$

(c) $X$ determines in $X(W) \subset M, n$ differential forms of degree 1 , $d w_{1}, \ldots, d w_{n}$

29. (a) $\left(T_{p} M\right)^{*}=$ dual space to $T_{p} M$

(b) For each $p \in X(W),\left\{d w_{1}(p), \ldots, d w_{n}(p)\right\}$ forms a basis for $\left(T_{p} M\right)^{*}$

(c) $\left\{d w_{1}(p), \ldots, d w_{n}(p)\right\}=$ dual basis to $\left\{d X(x) \cdot e_{1}, \ldots, d X(x) \cdot e_{n}\right\}$ 


\section{\#definition \#differential forms \#r-linear form}

30. (a) $M^{n}=$ a surface of dimension $n$

(b) $T_{p} M=$ tangent space to $M$ at $p$

(b) For each $p \in M$, the map $\psi: M \rightarrow A_{r}\left(T_{p} M\right)$ associates the $r$-linear form $\psi(p) \in A_{r}\left(T_{p} M\right)$

(c) $\psi=$ differential form of degree $r$ on $M$ 


\section{\#definition \#dual map \#linear map \#transpose}

31. (a) Let $V$ and $W$ be two vector spaces

(b) For each linear map $\psi: V \rightarrow W$ we define $\psi^{*}: V^{*} \rightarrow W^{*}$, by $\psi^{*}(f)(u)=f(\psi(u))$ for $f \in V^{*}, u \in W^{*}$

(c) $\psi^{*}=$ the dual map or transpose of $\psi$ 


\section{\#definition \# exterior product \#linear forms}

32. The exterior product of $\psi \in A_{r}(V)$ and $\xi \in A_{s}(V)$, is the map $\psi \wedge \xi \in A_{r+s}(V)$ defined by

$$
\psi \wedge \xi=\frac{(r+s) !}{r ! s !} A l t(\psi \bigotimes \xi)
$$




\section{\#definition \#n-linear form \#volume element}

33. (a) $V$ = vector space with inner product $\langle$,

(b) If $\left\{e_{1}, \ldots, e_{n}\right\}$ is an positive orthonormal basis for $V$

(c) For all $u_{1}, \ldots, u_{n} \in V \Rightarrow u_{j}=\sum_{i=1}^{n} a_{i j} e_{i}$ and $A=\left(a_{i j}\right)$

(d) Let us define $\psi\left(u_{1}, \ldots, u_{n}\right)=\operatorname{det} A$, called the volume element of V

34. (a) $\psi \in A_{n}(V)$

(b) $g=\left(\left\langle v_{i}, v_{j}\right\rangle\right)$ (The Gram matrix)

(c) $g=A^{t} \cdot A \Rightarrow \operatorname{det} g=(\operatorname{det} A)^{2}$

(d) $\psi\left(u_{1}, \ldots, u_{n}\right)= \pm \sqrt{\operatorname{det}\left(\left\langle v_{i}, v_{j}\right\rangle\right)}=(\operatorname{sign} o f \operatorname{det} A) \sqrt{\operatorname{det}\left(\left\langle v_{i}, v_{j}\right\rangle\right)}$ 


\section{\#definition \#permutation \#sign of a permutation \#transposition}

35. $P_{r}=$ set of all permutations of the set $\{1,2, \ldots, r\}$

36. $\sigma \in P_{r}$ is a transposition if there is $(i, j)$ in $\{1,2, \ldots, r\}$ that such $\sigma(i)=j, \sigma(j)=i$ and $\sigma(l)=l \forall l \neq i, j$

37. If $\sigma \in P_{r} \rightarrow \sigma=\beta_{1} \cdot \ldots \cdot \beta_{b}$, where $\beta_{1}, \ldots, \beta_{b}$ are transpositions

38. For all $\sigma \in P_{r}, \operatorname{sgn} \sigma=\operatorname{sign}$ of $\sigma$

39. $\operatorname{sgn} \sigma=1$ if $b$ is even, and $\operatorname{sgn} \sigma=-1$ if $b$ is odd 


\section{\#definition \#pullback \#r-linear form}

40. (a) Let $V$ and $W$ be two vector spaces

(b) For each $r$, the linear map $\psi: V \rightarrow W$ determines $\psi^{*}: A_{r}(W) \rightarrow$ $A_{r}(V)$ defined by

$$
\psi^{*}(\xi)\left(u_{1}, \ldots, u_{r}\right)=\xi\left(\psi\left(u_{1}\right), \ldots, \psi\left(u_{r}\right)\right)
$$

for $\xi \in A_{r}(W), u_{1}, \ldots, u_{r} \in V$

(c) $\psi^{*}(\xi)=$ the pullback of $\xi$ for the space $V$ through $\psi$ 


\section{\#definition $\# r$-form $\# r$-linear form}

41. A map $\psi \in L_{r}(V)$ is a $r$-linear form ou $r$-form if $\psi\left(u_{1}, \ldots, u_{r}\right)=0$, as long as there are $i \neq j$ such that $u_{i}=u_{j}$ 


\section{\#definition \#r-linear map}

42. (a) $V$ vetor space of dimension $n$

(b) A map $\psi: V \times \cdots \times V \rightarrow \mathbb{R}$ is said to be $r$-linear if $\psi$ is linear in each of its variables

43. $L_{r}(V)=$ real vector space of all $r$-linear maps

44. $L_{1}(V)=E^{*}$, the dual space to $E$ 


\section{\#definition \#tensor product}

45. The tensor product of $\psi \in L_{r}(V)$ and $\xi \in L_{s}(V)$ is the map $\psi \otimes \xi \in L_{r+s}(V)$ defined by

$$
\psi \otimes \xi\left(u_{1}, \ldots, u_{r}, v_{1}, \ldots, v_{s}\right)=\psi\left(u_{1}, \ldots, u_{r}\right) \cdot \xi\left(v_{1}, \ldots, v_{s}\right)
$$

for any $u_{1}, \ldots, u_{r}, v_{1}, \ldots, v_{s} \in V$

46. The tensor product is associative, but it is not commutative 


\section{\#determinant \#linear forms}

47. (a) If $\psi \in A_{n}(V)$ and $\left\{u_{1}, \ldots, u_{n}\right\}$ is any basis for $V$

(b) $\psi\left(w_{1}, \ldots, w_{n}\right)=\operatorname{det}\left(a_{i j}\right) \cdot \psi\left(u_{1}, \ldots, u_{n}\right) \forall u_{1}, \ldots, u_{n} \in V$, where $w_{i}=\sum a_{i j} u_{j}$ 
\#differentiable \#differential forms \# exterior product \#properties \#wedge product

48. (a) $(\beta \wedge \psi) \wedge \gamma=\beta \wedge(\psi \wedge \gamma)$

(b) $\beta \wedge \psi=(-1)^{r s} \psi \wedge \beta$

(c) $\beta \wedge(\psi+\gamma)=\beta \wedge \psi+\beta \wedge \gamma$

(d) $(\beta+\psi) \wedge \gamma=\beta \wedge \gamma+\psi \wedge \gamma$

(e) $(g \cdot \beta) \wedge(h \cdot \psi)=g h(\beta \wedge \psi)$, for any $g, h \in C^{\infty}(M)$ 
\#differentiable \#differential forms \#properties \#pullback \#smooth map

49. Let $f: M \rightarrow N$ and $h: N \rightarrow S$ be two smooth maps
(a) $f^{*}(\beta+\psi)=f^{*} \beta+f^{*} \psi$
(b) $f^{*}(\beta \wedge \psi)=f^{*} \beta \wedge f^{*} \psi$
(c) $f^{*}(h \cdot \psi)=f^{*} h \cdot f^{*} \psi$
(d) $(h \circ f)^{*} \psi=f^{*} h^{*} \psi$ 


\section{\#differential forms \#r-linear form \#vector space}

50. The set of differential forms of degree $r$ on $M$ is a real vector space.

51. A differential form of degree zero is a real function $\psi: M \rightarrow \mathbb{R}$ 
\# exterior product \#linear forms \#properties

52. $\psi \wedge(\xi+\eta)=\psi \wedge \xi+\psi \wedge \eta$ (distributive over addition)

53. $\psi \wedge(\xi \wedge \eta)=(\psi \wedge \xi) \wedge \eta($ associativity $)$

54. If $\psi, \xi \in V^{*} \Rightarrow \psi \wedge \xi=\psi \otimes \xi-\xi \otimes \psi$

55. $\psi \wedge \xi=-\xi \wedge \psi$

56. $\psi \wedge \psi=0$ 


\section{Open Invitation}

Please review this article, add content, co-author, and join the Open Mathematics Collaboration. Contact alvaroyucra@uft.edu.br.

\section{Open Science}

The latex file for this paper together with other supplementary files are available [5].

\section{Ethical conduct of research}

This original work was pre-registered under the OSF Preprints [6] following the structure from [4], please cite it accordingly [7]. This will ensure that researches are conducted with integrity and intellectual honesty at all times and by all means.

\section{Acknowledgement}

+ Center for Open Science https://www.cos.io

+ Open Science Framework https://osf.io

\section{References}

[1] Lee, John M. Introduction to Smooth Manifolds. New York: Springer, 2001.

[2] Lee, Jeffrey M. Manifolds and Differential Geometry. Graduate Studies in Mathematics. Vol. 107, 2009. 
[3] Hirsch, Morris W. Differential Topology. New York: Springer. Vol. 33, 1976.

[4] Lobo, Matheus P. "Open Mathematics Knowledge Base." OSF Preprints, 28 Jun. 2020. https://doi.org/10.31219/osf.io/evq8a

[5] Lobo, Matheus P. "Open Journal of Mathematics and Physics (OJMP)." OSF, 21 Apr. 2020. https://doi.org/10.17605/OSF.IO/6HZYP

[6] COS. Open Science Framework. https://osf.io

[7] Hancco, Alvaro J. Y. "Differential Forms (Open Mathematics Knowledge Base)." OSF Preprints, 19 July 2020. https://doi.org/10.31219/osf.io/g7uqb

\section{The Open Mathematics Collaboration}

Alvaro Julio Yucra Hancco (lead author, alvaroyucra@uft.edu.br) ${ }^{1}$ https://orcid.org/0000-0001-6280-8775

Matheus Pereira Lobo (mplobo@uft.edu.br) $)^{1,2}$ https://orcid.org/0000-0003-4554-1372

${ }^{1}$ Federal University of Northern Tocantins (Brazil) ${ }^{2}$ Universidade Aberta (UAb, Portugal) 